\section{Persistent hydronephrosis after pyeloplasty: Is it a true obstruction? The role of endourology}

\author{
Ermelinda Mele, Michele Innocenzi, Laura Del Prete and \\ Nicola Capozza*
}

Pediatric Urology Department, Bambino Gesù Children's Hospital and Research Institute, Rome, Italy

\section{Abstract}

Introduction: Anderson-Hynes pyeloplasty remains the gold standard in the treatment of ureteropelvic junction obstruction. The diagnostic criteria for defining the failure of pyeloplasty are not well-defined or even arbitrary. Likewise, the ideal treatment of persistent hydronephrosis after pyeloplasty is not well established. We tested an innovative endourological procedure, which simultaneously allows a diagnostic definition of failure and treatment when necessary.

Materials and methods: The endourological procedure was applied prospectively to 13 cases from 2006 to 2015. The mean hydronephrosis was $3 \mathrm{~cm}$ and all the patients showed an obstructive pattern at scintigraphie. Of these, only 2 patients had symptoms. The procedure consisted in the endoscopic calibration of the pyeloureteral junction. In case of confirmed persistent stenosis, the procedure continued with the high pressure dilation of the junction. The calibration/dilation procedure was carried out with a balloon catheter, previously used for high pressure dilation in the obstructive megaureter. In all patients, a ureteral stent was positioned for 6 - 8 weeks.

The patients were then followed up using ultrasound and renoscintigraphie.

Results: According to the endoscopic balloon procedure, anastomosic stenosis was confirmed in 3 cases, treated with high pressure dilation during the same procedure. In 10 cases no stenosis was found and we followed-up these patients with periodic ultrasound and scintigraphie.

Conclusion: The calibration/dilation of the pyeloureteral junction represents in our opinion a useful diagnostic and therapeutic tool that allows to limit the repetition of open surgery only to symptomatic cases and those non-responders to endoscopic treatment.

\section{More Information}

*Address for Correspondence: Nicola Capozza, Pediatric Urology Department, Bambino Gesù Children's Hospital and Research Institute, Piazza S. Onofrio, 400165, Rome, Italy, Tel: +39.0668592402;

Fax: +39.06.6859.2849;

Email: nicola.capozza@opbg.net

Submitted: 10 December 2019

Approved: 09 April 2020

Published: 13 April 2020

How to cite this article: Mele E, Innocenzi M, Del Prete L, Capozza N. Persistent hydronephrosis after pyeloplasty: Is it a true obstruction? The role of endourology. J Clini Nephrol. 2020; 4: 016-019.

DOI: 10.29328/journal.jcn.1001052

Copyright: ( 2020 Mele E, et al. This is an open access article distributed under the Creative Commons Attribution License, which permits unrestricted use, distribution, and reproduction in any medium, provided the original work is properly cited.

Keywords: Persistent obstructive hydronephrosis; Ballon dilation; Pyeloplasty; Renal scintigraphy

Check for updates

OPEN ACCESS

\section{Introduction}

Dismembered pyeloplasty remains the gold standard in the treatment of ureteropelvic junction (UPJ) obstruction. Although the success rate is more than $80 \%$ [1], the reasons underlying persistent hydronephrosis after pyeloplasty and its management remain controversial. The causes of failure are not clear. Some authors have hypothesized risk factors such as young age at operation, the short duration of ureteral stenting, or the presence of hypoplastic ureter [2]. Moreover , the diagnostic criteria for defining the failure of pyeloplasty are not well-defined or even arbitrary. The definition of failure is based on the persistent or increased pyelic dilation and scintigraphic results (persistence of obstructive pattern, decreased function of the affected kidney). Likewise, there is no consensus on the type of treatment for failed of pyeloplasty; possibilities include surgery (open or laparoscopic) or endoscopy.
The aim of our study was the evaluation of an innovative endourological procedure for failed pyeloplasty, which simultaneously allows a diagnostic definition and treatment if necessary.

\section{Material and methods}

We considered for our study 13 patients with persistent hydronephrosis after dismembered pyeloplasty, treated consecutively between January 2006 and December 2015. Only patients with persistent obstructive hydronephrosis at one year follow-up were included in the present study.

We excluded patients with hydronephrosis due to crossing vessels. The pyeloplasty technique used in all patients was OPRAP, as described in previous studies [3]. The initial follow-up after pyeloplasty (Follow-up 1) consisted of ultrasound at 3 and 6 months and MAG3-scintigraphie at 9 - 12 months. Persistent hydronephrosis was defined as 
dilation unchanged or worsened with a persistent obstructive pattern at scintigraphie. All the 13 patients with persistent hydronephrosis underwent retrograde pyelography and ureteral calibration/dilation with a balloon catheter (Passeo-18).

The balloon catheter, originally designed for angioplasty, is currently used by our team for the treatment of obstructive megaureter [4]. It has a caliber of 3,8 French; the balloon can be inflated with contrast medium up to a diameter of $4 \mathrm{~mm}$ (patients 6 - 24 months of age) to $6 \mathrm{~mm}$ (over 2 years) and up to a pressure of 12 atmospheres.

The patients in this study underwent cystoscopy under general anesthesia, using an 8-9.8 Ch cystoscope. The catheter was inserted through the ureter into the renal pelvis, the balloon was inflated with contrast medium until it reached a diameter of $4-6 \mathrm{~mm}$. The catheter was then withdrawn gradually under fluoroscopic guidance. Where it was possible to withdraw the catheter without difficulty, patency of the ureteropelvic junction was demonstrated; the diameter was of $4-6 \mathrm{~mm}$. In the case of difficulty in advancing the balloon across the UPJ or where it was not possible to advance it, real persistence of stenosis was shown. In such cases, the balloon was deflated and repositioned at the UPJ level, and high pressure dilation was employed at $12 \mathrm{~atm}$ for $5 \mathrm{~min}$. At the end of the dilation procedure, a double J ureteral stent was positioned for 6 weeks. In the case of simple calibration without dilation, no stent was required. All patients with persistent hydronephrosis were again followed-up (Follow-up 2) with ultrasound at 3 and 6 months and MAG3-scintigraphie (scinti 2) at 12 months; thereafter, with ultrasound yearly.

\section{Results}

Over a period of 9 years, 487 consecutive pyeloplasties were performed with a success rate of $97.4 \%$. Prenatal or perinatal diagnosis was documented in all patients. The indications at surgery were mean hydronephrosis of $3.5 \mathrm{~cm}$ (range $2.5 \mathrm{~cm}-7.5 \mathrm{~cm}$ ) and an obstructive pattern at nuclear MAG3 renogram. Median age at surgery was 21 months (range 6 - 84). Of our patients, 13 children (2.6\%) failed initial procedures, and presented within 10 to 15 months (mean 12) postoperatively.

The average follow-up was 36.8 months $(24-96)$.

Of the 13 patients with persistent hydronephrosis, all were found to have a pre-pyeloplasty dilation mean of more than $3 \mathrm{~cm}$ and an obstructive pattern on scintigraphie. In one case, reduction of split renal function was observed. Two patients showed symptoms (recurrent flank pain). The endoscopic procedure observed (Table 1) anastomotic stenosis in 3 cases (Figure 1a). This ring was dilated with the balloon catheter for 5 minutes at 12 atm and the stenosis was resolved (Figure 1b). The procedure was easy, well tolerated, without any technical difficult.
In 10 patients, no stenosis was found during the endoscopic procedure (Figure 2). No complications were seen intra or postoperatively and no recurrence in the structure after balloon dilation has seen.

At Follow-up 2, split renal function remained stable in all patients (including the one with the initial loss of function); the diuretic test showed an improvement in urine drainage; ultrasonography documented a progressive reduction in pelvic dilation, but not of calices. The 2 symptomatic patients continued to complain of flank pain, although they resulted

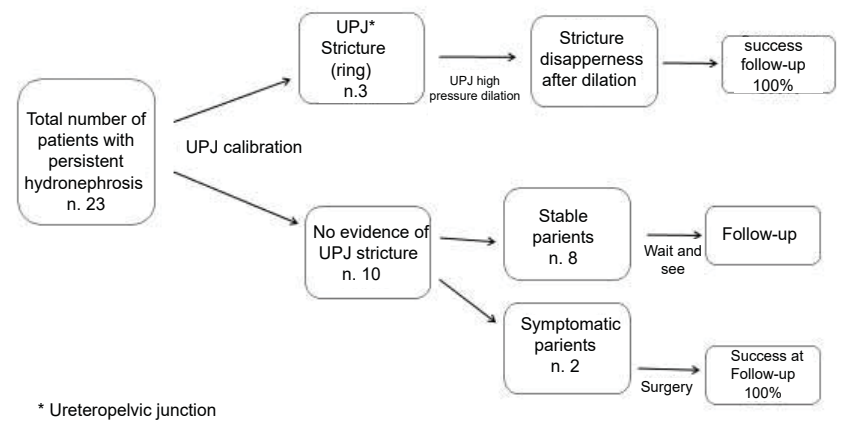

Table 1: Patients Mnagement.

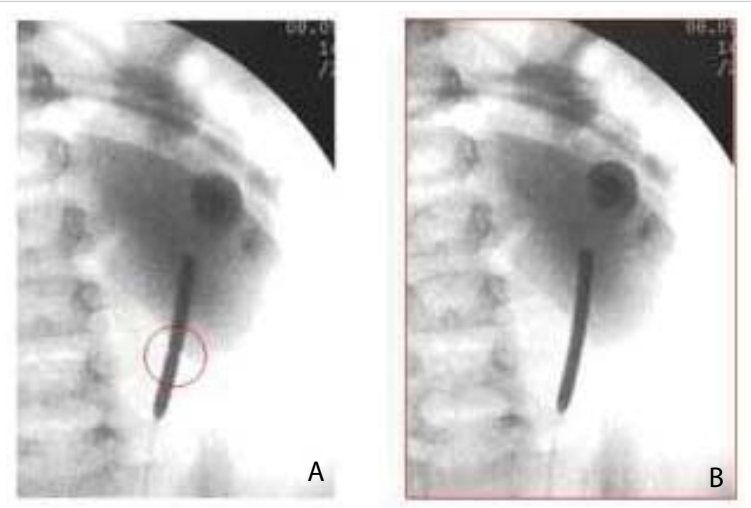

Figure 1: A: Stenosis of pyeloureteral anastomosis in red circle. B: Resolution of stenosis after ballon dilation.

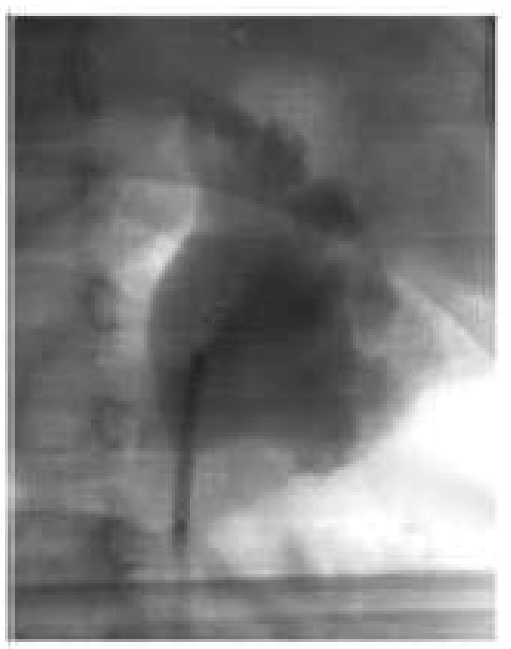

Figure 2: Endoscopic evidence of absence of pyelopureteral stenosis. 
"not obstructed" at balloon calibration. They were found to have decreased split renal function at scinti 2. For this reason, they underwent a further pyeloplasty, with the operative finding of scar tissue compressing the UPJ.

\section{Discussion}

Dismembered pyeloplasty is considered the treatment of choice in cases of obstructive hydronephrosis, with a success rate of about 98\% [1]. The literature offers no definitive conclusions regarding the causes of failure $[5,6]$. Of the possible risk factors, some authors suggest young age at intervention (less than 6 months) [6] and urinary leakage as the initial event leading to scar formation and fibrosis around the UPJ $[7,8]$. The incidence of crossing vessels associated with obstructive hydronephrosis is reported as being between $11 \%$ and $49 \%$ and is responsible for $10 \%$ of failed pyeloplasty owing to the lack of recognition of this condition at the first operation [5]. Baniel et al suggest that persistent obstruction can be attributed to inflammatory edema consequent to surgical manipulation [9]. Other risk factors could be a very dilated pelvis, insufficiently reduced at the surgical procedure, and the possibility of urinary filtration with secondary fibrosis around the anastomosis [8]. For these reasons, the authors stress the importance of the correct positioning of a stent with prolonged drainage to prevent filtration and subsequent obstruction. In fact, redundancy of the pelvis, hypoplasia of the ureter or inadequate spatulation of the ureter during intervention or any kinking may result in leakage of urine. This can lead to potential local inflammation and recurrence of stenosis $[2,10]$.

Regarding the management of persistent/recurrent hydronephrosis, opinions differ among researchers. Nicholls et al. suggest endopyelotomy for secondary symptomatic hydronephrosis in older children [11]. Figureenshau reports a success rate of $91 \%$ with endopyelotomy for secondary obstructions of the UPJ [12]. Kinn stresses the importance of the symptoms, in the absence of which hydronephrosis in adults should be considered a benign condition, to be managed conservatively. On the contrary, the same author suggest that the presence of symptoms is an indication for surgery [13]. Open surgery is also preferred by Thomas, et al. in view of the poor results of endoscopic dilation [5].

In our series, particular attention was paid to the abovementioned risk factors. As far as possible the same surgical technique was employed, which included prolonged postoperative drainage. The search for crossing vessels was meticulous and where they were identified the patients were excluded from the series. Interestingly, in 3 cases, the initial pelvic dilation was greater than the average of our overall series. The large dimensions of the pelvis could then be the main risk factor for persistent hydronephrosis after pyeloplasty. In our cases, the endourological procedure allowed confirmation that, in the majority of cases (10/13), there was no real recurrence of stenosis. There was instead a residual hypotonia of the pelvis and especially of the calices (already very dilated at the start), which were responsible for the persistence of the "apparently obstructive" pattern on scintigraphie. The ultrasound at Follow-up 2 confirmed a trend of the reduction of the pelvis, whereas the calices, for obvious anatomical reasons, tended to remain dilated. A further confirmation of the hypothesis that no real stenosis exists can be seen from the stability of the split renal function and the improvement (spontaneous, with no dilation procedure) of urinary drainage at scintigraphie 2 .

The calibration of the UPJ with a balloon catheter, proposed here, is essentially a diagnostic procedure, which excludes true recurrence of the obstruction in the majority of cases. In a few cases (3/13), where true stenosis was documented, the procedure also became therapeutic, with the use of high pressure dilation. One potential limitation of the endourological procedure is that it can fail to identify extrinsic compression of the UPJ by scar tissue, as a consequence of the initial operation. In this instance, the balloon catheter fails to reveal a stenosis. Clinical follow-up is essential in this situation. The 2 cases in our series with extrinsic compression, despite having a normal caliber at endoscopy and a stable split renal function, were both symptomatic and therefore had to undergo surgery.

\section{Conclusion}

Dismembered pyeloplasty remains the gold standard for treating obstructive hydronephrosis, achieving a very high success rate. In cases of failure, the endourologic calibration/ dilation of the UPJ is a minimally invasive procedure, simple, safe and with no potential risks and complications for the patients. It can be considered both as a useful diagnostic tool and as a first therapeutic approach in cases of persistent hydronephrosis after pyeloplasty. The causes of failed pyeloplasty are not completely clear, in most cases the persistence of hydronephrosis is due to prolonged hypotonia of the pelvis rather than to recurrence of stenosis. In the absence of symptoms, the assessment of an adequate caliber of the UPJ and stability of split renal function are sufficient reasons to avoid further surgical procedures.

\section{References}

1. Salem YH, Majd M, Rushton HG, Belman AB. Outcome analysis of pediatric pyeloplasty as a function of patient age, presentation and differential renal function. J Urol. 1995; 154: 1889-1893.

PubMed: https://www.ncbi.nlm.nih.gov/pubmed/7563377

2. Braga LH, Lorenzo AJ, Bägli DJ, Keays M, Farhat WA, et al. Risk factors for recurrent ureteropelvic junction obstruction after open pyeloplasty in a large pediatric cohort. J Urol. 2008; 180:1684-1688. PubMed: https://www.ncbi.nlm.nih.gov/pubmed/18708226

3. Caione P, Lais A, Nappo SG. One-Port Retroperitoneoscopic Assisted Pyeloplasty Versus Open Dismembered Pyeloplasty in Young Children: Preliminary Experience. J Urol. 2010; 184:2109-2115.

PubMed: https://www.ncbi.nlm.nih.gov/pubmed/20851429 
4. Torino G, Collura G, Mele E, Garganese MC, Capozza N. Severe primary obstructive megaureter in the first year of life: preliminary experience with endoscopic balloon dilation. J Endourol. 2012; 26: 325-329.

PubMed: https://www.ncbi.nlm.nih.gov/pubmed/22050492

5. Thomas JC, DeMarco RT, Donohoe JM, Adams MC, Pope JC, et al. Management of the failed pyelopasty: a contemporary review. J Urol. 2005; 174: 2363-2366.

PubMed: https://www.ncbi.nlm.nih.gov/pubmed/16280844

6. Lim DJ, Walker RD. Management of the failed pyeloplasty. J Urol. 1996; 156:738-740.

PubMed: https://www.ncbi.nlm.nih.gov/pubmed/8683772

7. Persky L, McDouglas WS, Kedia K. Management of initial pyeloplasty failure. J Urol. 1981; 125:695.

8. Rohrmann D, Snyder HM, Duckett JW, Canning DA, Zderic SA. The operative management of recurrent ureteropelvic junction obstruction. J Urol. 1997; 158:1257-1259.

PubMed: https://www.ncbi.nlm.nih.gov/pubmed/9258188

9. Baniel J, Livne PM, Savir A, Gillon G, Servadio C. Dismembered pyeloplasty in children with and without stents. Eur Urol. 1996; 30: 400-402. PubMed: https://www.ncbi.nlm.nih.gov/pubmed/8931977
10. Smith KE, Holmes N, Lieb JI, Mandell J, Baskin LS, et al. Stended versus nonstended pediatric pyeloplasty: a modern series and review of the literature. J Urol. 2002; 168:1127-1130.

PubMed: https://www.ncbi.nlm.nih.gov/pubmed/12187251

11. Nicholls G, Hrouda D, Kellett MJ, Duffy PG. Endopyelotomy in the symptomatic older child. BJU International. 2001; 87: 525-527. PubMed: https://www.ncbi.nlm.nih.gov/pubmed/11298049

12. Figureenshau RS, Clayman RV. Endourologic options for management of ureteropelvic junction obstruction in the pediatric patient. Urol Clin North Am. 1998; 25:199-209.

PubMed: https://www.ncbi.nlm.nih.gov/pubmed/9633575

13. Kinn AC. Ureteropelvic junction obstruction: long-term followup of adults with and without surgical treatment. J Urol 2000; 164: 652-656. PubMed: https://www.ncbi.nlm.nih.gov/pubmed/10953119

14. Parente A, Angulo JM, Romero RM, Rivas S, Burgos L, et al. Managment of ureteropelvic junction obstruction with high-pressure ballon dilatation: long-term outcome in 50 children under 18 months of age. Urol. 2013; 82: 1138-1143.

PubMed: https://www.ncbi.nlm.nih.gov/pubmed/23992967 